\title{
The Possibility of Applying Spectral Redundancy in Coherent DWDM Systems for Increasing the Data Transmission Rate and Decreasing Nonlinear Effects and Double Rayleigh Scattering with Great Security of Data
}

\author{
Alexander Nekuchaev, Sergey Shuteev \\ Moscow State University, Moscow, Russia \\ Email: orovich@rande1.ru \\ Received 26 November 2015; accepted 19 February 2016; published 22 February 2016 \\ Copyright (C) 2016 by authors and Scientific Research Publishing Inc. \\ This work is licensed under the Creative Commons Attribution International License (CC BY). \\ http://creativecommons.org/licenses/by/4.0/

(c) (i) Open Access

\begin{abstract}
A new method coherent DWDM system has been proposed. Standard method uses 90 wavelengths with channel capacity $100 \mathrm{G}$, so total capacity amounts to 9 Terabit, optical power is P. New "channel super" (CS) consists of 15 wavelengths, number of CS is 6, so total number of wavelengths is 90 too, total capacity is 6 Terabit, but optical power is 4/15P. Over ULH and EULH markets, standard approach will be forced to reduce the total capacity to 2.4 Terabit with optical power $4 / 15 \mathrm{P}$ as in proposed method. But the new can reach 6 Terabit with the same optical power 4/15P.
\end{abstract}

\section{Keywords}

Coherent Communications, Optical Security and Encryption, Fiber Optics Links and Subsystems

\section{Introduction}

The idea to use spectral redundancy was patented by the Department of Physics, Moscow State University [1]. The development of this method was later suggested [2] with principal improvement-instead of wavelengths, pilots and slaves were proposed. In the present work, a new method of data transmission in coherent DWDM systems is proposed. The existing method, e.g., uses 90 wavelengths $100 \mathrm{G}$ with total capacity 9 Terabit/sec 
with average power P. In the new method $6 \mathrm{CS}$, every with 15 wavelengths is used providing total capacity 6 Terabit/sec with an average power 4/15P. The cross modulation and double Rayleigh scattering are significantly decreased owing to the uniform distribution of power overtime at different wavelengths. The time redundancy (forward error correction (FEC)) is about 20\% - 25\% and allows one to achieve a net coding enhancement of about $10^{-15} \mathrm{~dB}$ by detecting and removing deletions and errors simultaneously. Security of data amounts to $90 ! \approx$ $10^{135}$ combinations. If any laser destroys, BER increases from $10^{-15}$ to $10^{-12}$ without interruption.

\section{Description of Method}

The essence of the method is explained in Figure 1. The time is divided into cycles, each of which is equal to 4 microcycles. Eight wavelengths $\mathrm{P}_{\mathrm{ij}}$ are called pilots (the duration of each wavelength is equal to one cycle) each of which corresponds to a three bit chip: $000(\mathrm{i}=1), 001(\mathrm{i}=2)$, and so on to $111(\mathrm{i}=8)$. $\mathrm{J}=1-4$ according 4 QPSK positions. The pilots are shown in Figure 1 by solid lines. Waves $S_{\mathrm{ijk}}$ are called slaves and shown in the figure by dashed lines. Slave $S_{1 j k}$ means that, in a given microcycle, the content separated from the current content by one microcycle is transmitted back; $S_{2 j k}$ is separated by 2 microcycles, and $S_{3 j k}$ is separated by 3 microcycles ( $\mathrm{j}=1-4$ according 4 QPSK positions, $\mathrm{k}$ is a degenerated factor). Total number of slaves is equal to 7 , because there are $S_{1 j 1}, S_{1 j 2}, S_{2 j 1}, S_{2 j 2}, S_{3 j 1}, S_{3 j 2}$ and $S_{3 j 3}$. Every slave is sent in sequence, for example as shown on Figure 1, where combination $S_{3 j 1}, S_{3 j 2}, S_{3 j 3}$ is necessity. Total number of wavelengths in one "channel super" is equal to 15. Total number of "channel super” is equal to 6. Total number of wavelengths is equal to 90 . Before optical MUX with 90 wavelengths optical power of every channel goes through on/off optical switch, which operates with at least micro cycle frequency. Duration of “on” open state is four micro cycles; duration of "off” closed state is zero micro cycle and any more. General scheme is shown on Figure 2. Transmitter in every “channel super” works as following both in Ox and Oy polarization (see Figure 3).

Every pilot carries 3 bits as colour and 2 bits as QPSK, together 5 bits. In any time there are 4 different wavelengths. During cycle $4 \times 5=20$ bits with 15 different wavelengths are transmitted in proposed method against 30 bits with 15 different wavelengths as usually. So total capacity will be 6 times with 15 or 90 different wavelengths, which corresponds to $9 \times(20 / 30)=6$ Terabit/sec opposite to 9.0 Terabit/sec in usual method, but power will be 15/4 = 3.75 times less $10 \mathrm{lg} 3.75=5.74 \mathrm{~dB}$. Over ULH and EULH market nonlinear effects [3] reduce total capacity strongly as shown in Table 1 [4].

So for reach more than approximately $5000 \mathrm{~km}$ provider will turn off a lot of wavelengths (because in nonlinear optics power per channel reduces as $\mathrm{P}$ in square when the number of channels is big, opposite as $\mathrm{P}$ in linear optics [3] when the number of channels is small), for example in 15/4 times, reducing total power and capacity (see Table 1 [4]). REAL capacity will be $9 \times 4 / 15=2.4$ Terabit/sec. In new method power will be same but REAL capacity will be again 6 Terabit/sec with DRS $5.74 \mathrm{~dB}$ less! Very interesting that there are combinations, when in both approaches transmitter sends absolutely the same, but total capacity differs 2.5 times. Note, that submarine systems cannot be upgraded, so in usual methods double power $2 \mathrm{P}$ for two polarizations may be challenge, but our budget is big enough, because $2 \times 4 / 15 \mathrm{P}=8 / 15 \mathrm{P}$.

Is such transmitter possible? Answer is easy-yes!

Is corresponding receiver possible? Answer is extremely difficult. We propose next construction shown on Figure 4 (for simplicity clock or cycle is sent over separate wavelength for all "channel super" in every fiber for cable altogether). After optical DMX (90 channels) polarization beam splitter divides signal to Ox and Oy. Then Y splitter divides optical signal to amplitude and phase analyzer devices.

The amplitude analyzer device works without coherent techniques. Splitter divides fiber on four direction, every with standard IM-DD (Intensity Modulation with Direct Detection) technique. Every detector works with equal clock, but with micro cycle shift and every cycle has 1 or 0 on exit. Then four exits are added providing "amplitude pool” digital signal, which will be upstairs and downstairs. Then special amplitude decoder (with FEC) calculates exactly micro cycle with true 3 bit in true micro cycle.

There are two types of time redundancy. First is for frame synchronization. Every frame consists of 20 micro cycles. First micro cycle we send nothing, without any color. Second and third are useful load, fourth-control summer, which is 5 bits opposite to in second micro cycle. So the total redundancy amounts to $10 \%$ and it guarantees exact and quick calculation of contents 1 - 4 micro cycles. After this "start-up” FEC with 10\% - 15\% redundancy begins "step by step” calculation in past and future may be with Viterby, because our channel is a channel with memory. The total redundancy is $20 \%-25 \%$. 


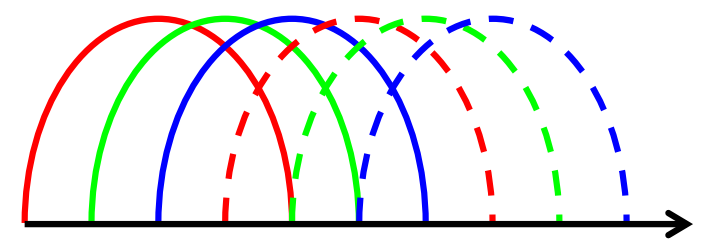

Need: $\quad \mathbf{P}_{1 \mathrm{j}} \quad \mathbf{P}_{2 \mathrm{j}} \quad \mathbf{P}_{3 \mathrm{j}} \quad \mathbf{P}_{\mathbf{1 j}} \quad \mathbf{P}_{2 \mathrm{j}} \quad \mathbf{P}_{3 \mathrm{j}}$

Sent: $\quad \mathbf{P}_{1 \mathrm{j}} \quad \mathbf{P}_{2 \mathrm{j}} \quad \mathbf{P}_{3 \mathrm{j}} \quad \mathbf{S}_{3 \mathbf{j} 1} \mathbf{S}_{3 \mathrm{j} 2} \quad \mathbf{S}_{3 \mathrm{j} 3}$

Figure 1. Content is data only with pilots, real transmitted data are pilots and slaves.

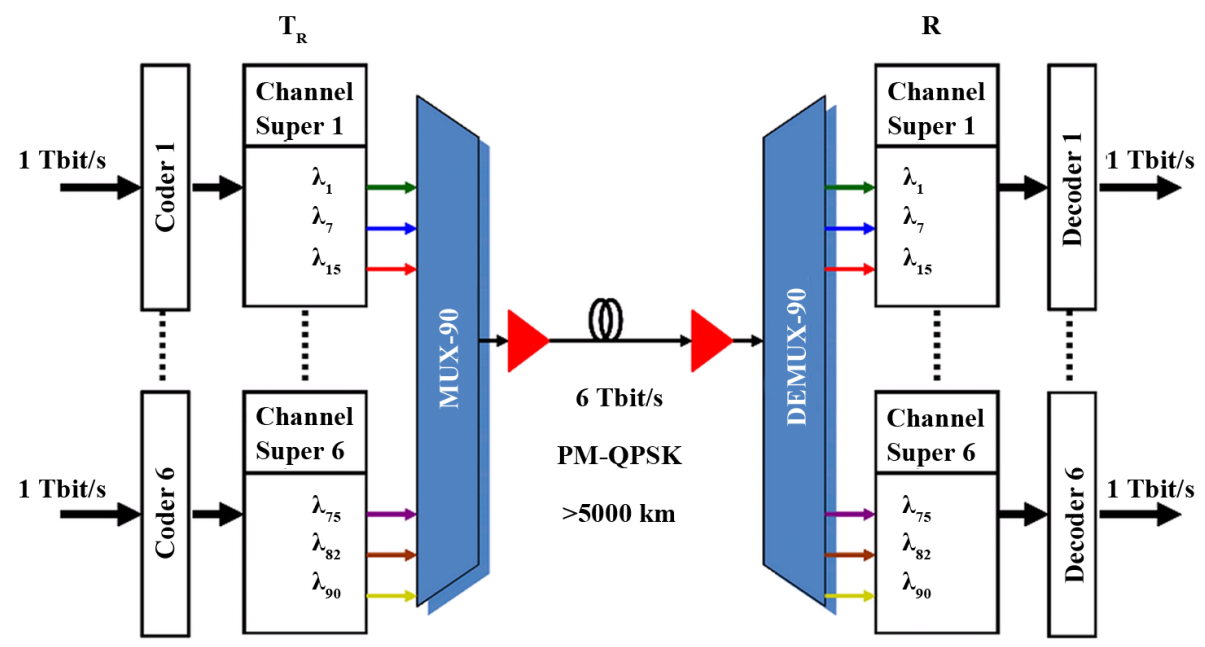

Figure 2. General scheme.

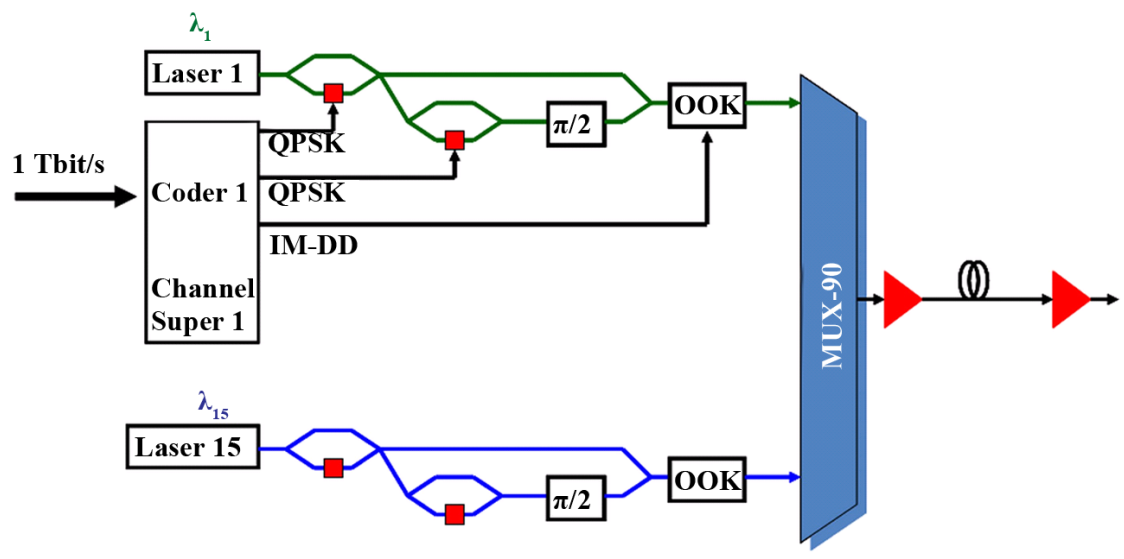

Figure 3. Transmitter in every “channel super”.

Table 1. Increasing spectral efficiency with higher order modulation.

\begin{tabular}{ccc}
\hline Modulation & Range & Performance in range C (flexible frequency) \\
\hline PM-BPSK & $5000 \mathrm{~km}$ & 5 Terabit/sec \\
PM-QPSK & $3000 \mathrm{~km}$ & 10 Terabit/sec \\
PM-8QAM & $1500 \mathrm{~km}$ & 15 Terabit/sec \\
PM-16QAM & $700 \mathrm{~km}$ & 20 Terabit/sec \\
PM-32QAM & $350 \mathrm{~km}$ & 30 Terabit/sec \\
\hline
\end{tabular}




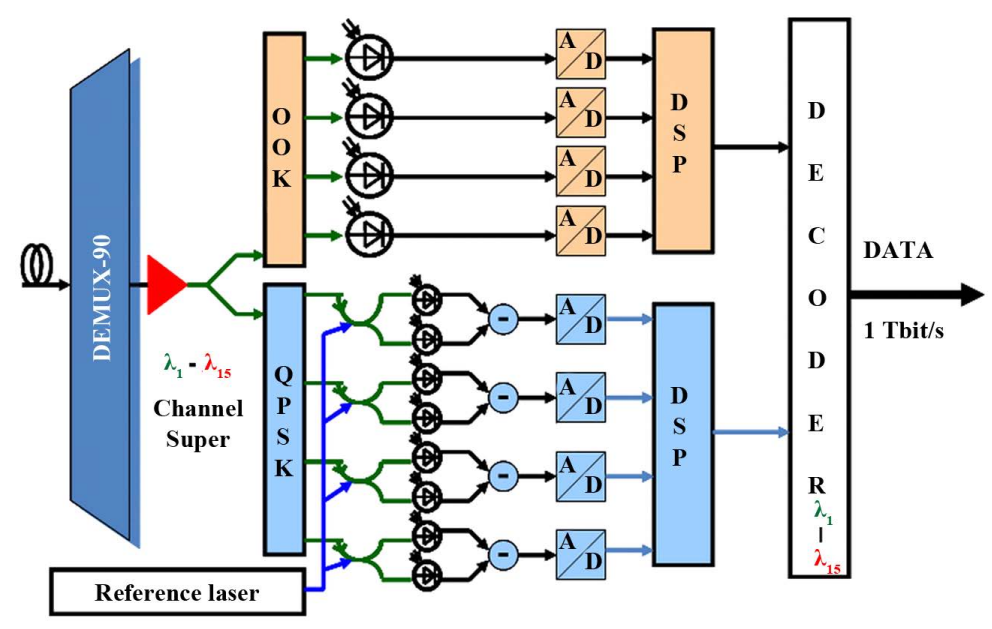

Figure 4. Receiver in every “channel super”.

Phase analyzer device works with coherent technique. Reference signal and data signal are sent to four phase detectors, every of which works during cycle with shifting micro cycle. Every such QPSK (of course not differential) detector will receive standard signal (SS) and non standard signal (NSS). SS will be checked by FEC. But we believe that NSS has useful information too. So special phase decoder with FEC will analyse both SS and NSS. Special amplitude decoder and special phase decoder tells each other about amplitude and phase pool in the same time and send out 3 bits and 2 bits in sequence.

Note that there are special mistakes (SM) and real mistakes (RM). SM is the result of decoding real energy (sent by transmitter) in both amplitude and phase decoders. RM is the result of decoding real noise in both amplitude and phase decoders. Many RM (single and may be even more) are deleted without redundancy, because they are in contradiction with pause or received energy as pool. These situations have been investigated in the Institute for Information Transmission Problems [5].

Let us consider several situations on receiver in details after transmitter sent next arbitrary frames:

\begin{tabular}{cccccccccccccc}
\hline № & $\ldots 20$ & 1 & 2 & 3 & 4 & 5 & $\ldots$ & 19 & 20 & 1 & 2 & 3 & 4 \\
\hline$\lambda$ & & - & $\mathrm{P}_{1 \mathrm{j}}$ & $\mathrm{P}_{2 \mathrm{j}}$ & $\mathrm{P}_{-1-\mathrm{j}}$ & $\mathrm{S}_{3 j 1}$ & $\ldots$ & & & - & $\mathrm{P}_{8 \mathrm{j}}$ & $\mathrm{P}_{7 \mathrm{j}}$ & $\mathrm{P}_{-8-\mathrm{j}}$ \\
\hline
\end{tabular}

Received data on wavelength P1 in amplitude decoders will be

\begin{tabular}{cccccccccccc}
\hline № & 16 & 17 & 18 & 19 & 20 & 1 & 2 & 3 & 4 & 5 \\
\hline$\lambda$ & - & - & $\mathrm{P}_{1 \mathrm{j}}$ & $\mathrm{P}_{1 \mathrm{j}}$ & $\mathrm{P}_{1 \mathrm{j}}$ & $\mathrm{P}_{1 \mathrm{j}}$ & $\mathrm{P}_{1 \mathrm{j}}$ & $\mathrm{P}_{1 \mathrm{j}}$ & $\mathrm{P}_{1 \mathrm{j}}$ & - \\
\hline
\end{tabular}

Received data on wavelength P1 in phase decoders will be

\begin{tabular}{llllllllll}
\hline$?$ & $?$ & $?$ & & $?$ & & $?$ & $\mathrm{P}_{1 \mathrm{j}}$ & $?$ & $?$ \\
\hline
\end{tabular}

Received data on wavelength P2-P8, S1-S7 in amplitude decoders may be

$$
20
$$$$
1
$$$$
2
$$

$$
\mathrm{P}_{2}-\mathrm{P}_{8}, \mathrm{~S}_{1}-\mathrm{S}_{7}
$$

Received data on wavelength P1 in phase decoders may be

20

$\mathrm{P}_{2}-\mathrm{P}_{8}, \mathrm{~S}_{1}-\mathrm{S}_{7}$

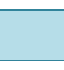


Thanks to the pause $\mathrm{P}_{1 \mathrm{j}}$ data will be recovered absolutely exactly and quickly in amplitude decoder. P2-P8, S1-S7 will be deleted without redundancy as impossible. $\mathrm{P}_{1 \mathrm{j}}$ data in phase decoder will be recovered using FEC and control summer P-1-j. So our "soft decision" means that we can increase sensitivity in photo detectors in order to improve operation of amplitude detectors without danger of $\mathrm{P}_{2}-\mathrm{P}_{8}, \mathrm{~S}_{1}-\mathrm{S}_{7}$.

Then step by step FEC with redundancy $10 \%-15 \%$ will recover in future microcycle $5,6, \ldots$ and in past 20 , $19,18, \ldots$ These both data will meet without contradiction.

In addition security of data is extremely high since illegitimate user will be forced to find 15 wavelengths (they are virtual, not adjacent obligatory) among 90, then find pilots and slavers, than status of every pilot and every slave. The number of possible combinations is equal to $90 ! \approx 10^{135}$, if illegitimate user can on physical level copy 90 channels to memory.

If reach is more than $5000 \mathrm{~km}$ significantly, BPSK will be used instead of QPSK. Than over on clock 15 channels have 15 bits, but we 3 due to color +1 due to phase $=4$ bit/symbol. During one clock we send $4 \times 4=$ 16 bit/clock and our power and DRS [6] [7] will be 15/4 = 3.75 times less!

If reach is less than $5000 \mathrm{~km}(4000-5000 \mathrm{~km})$ the number of wavelengths in CS reduced for example 4 pilots +7 slaves $=11$ wavelengths instead of 8 pilots +7 slaves $=15$ wavelengths. Then 88 wavelengths in usual method in theory transmit 8.8 Terabit/sec, but in new 6.4 Terabit/sec, because in every CS every symbol transmits 2 bits due to color and 2 due to QPSK, number of $\mathrm{CS}=8$. The importance of nonlinear optics remains great because our total power $11 / 4=2.75$ times less or $10 \lg 2.75=4.4 \mathrm{~dB}$.

If reach amounts to $3000-4000 \mathrm{~km}$ then CS may be 2 pilots and 1 slave. Every symbol transmits 1 bit due to color and 2 bits due to QPSK. Then the total capacity will be 9 .

Terabit/sec in both approaches, but in new method total power will be $3 / 2=1.5$ times less, number of CS amounts to $30(30 \times 3=90)$. The importance of nonlinear optics is less of course, but not negligible.

If any laser destroys, BER increases from 10 - 15 to 10 - 12 without interruption. Slave $S_{33}$ begins to work instead of destroyed and if $S_{31}, S_{32}, S_{33}$ are necessary together, and then instead of $S_{33}$ we send pause and FEC recover two QPSK bits as deletion.

\section{Conclusions}

New method of data transmission has been proposed. In usual methods, only one symbol from some varieties is sent and only one symbol will be received-true or false. In new method, only one symbol from some varieties is sent and A LOT of symbol will be received-true or false. Then amplitude analysis provides true color and 3 bits and phase analysis provides true 2 bits.

Reducing DRS $(15 / 4=5.74 \mathrm{~dB})$ results in increasing reach of about $1000 \mathrm{~km}$. Over ULH and EULH markets, expected profit of transmission data rate is 2.5 - 3 times more, for example for under sea world. Security of data is extremely high (for existed and future terrestrial market, for any reach-EULH, ULH, long haul and so on). If any laser destroys, BER increases from 10 - 15 to 10 - 12 without interruption.

\section{References}

[1] Nekuchaev, A.O. and Yusupaliev, U. (2000) RF Inventor’s Certificate No. 2161374 (MGU, Fizicheskii Fakul’tet).

[2] Nekuchaev, A.O. and Shuteev, S.A. (2014) The Possibility of Applying Spectral Redundancy in DWDM Systems on Existing Long-Distance FOCLs for Increasing the Data Transmission Rate and Decreasing Nonlinear Effects and Double Rayleigh Scattering without Changes in the Communication Chanel. Optics and Spectroscopy, 116, 513-515. http://dx.doi.org/10.1134/S0030400X14040195

[3] Agraval, G.P. (2012) Nonlinear Fiber Optics, Fifth Edition (Optics and Photonics). Academic Press, Oxford, UK, Waltham, MA, USA, 648.

[4] http://www.infinera.com/resources/white-papers/

[5] Zhilin, I., Rybin, P. and Zyablov, V. (2015) High-Rate Codes for High-Reliability Data Transmission. IEEE International Symposium on Information Theory (ISIT 2015), Hong Kong, 14-19 June 2015, 256-260. http://www.isit2015.org/

[6] Isoe, G.M., Muguro, K.M. and Waswa, D.W. (2013) Noise Figure Analysis of Distributed Fibre Raman Amplifier. International Journal of Scientific \& Technology Research, 2, 2277-8616. http://www.ijstr.org/final-print/nov2013/Noise-Figure-Analysis-Of-Distributed-Fibre-Raman-Amplifier.pdf 
[7] Cai, Y., Cai, J.-X., Davidson, C.R., Foursa, D.G., Lucero, A.J., Sinkin, O.V., Sun, Y., Pilipetskii, A.N., Mohs, G. and Bergano, N. (2011) Ultra-Long-Haul WDM Transmission with High Spectral Efficiency. IEICE Transactions on Communications, E94.B, 392-399. http://dx.doi.org/10.1587/transcom.E94.B.392

http://search.ieice.org/bin/pdf_link.php?abst=\&category=B\&fname=e94-b_2_392\&lang=E\&year=2011 